\title{
Protect, care and conserve
}

At the forefront of preventative and minimally invasive restorative dentistry VOCO are passionate to help dental professionals protect, care and conserve original tooth structure.

To further this aim, ensuring that patients teeth are kept strong and functional for life, VOCO have developed Profluorid Varnish, which is suitable for a wide variety of indications and gives effective relief from the common problem of hypersensitive teeth. The white, transparent varnish is specifically designed for the treatment of exposed root surfaces, cervical defects, damaged enamel and carious lesions as well as for sealing the dentinal tubules following cavity preparation. Thanks to its sodium

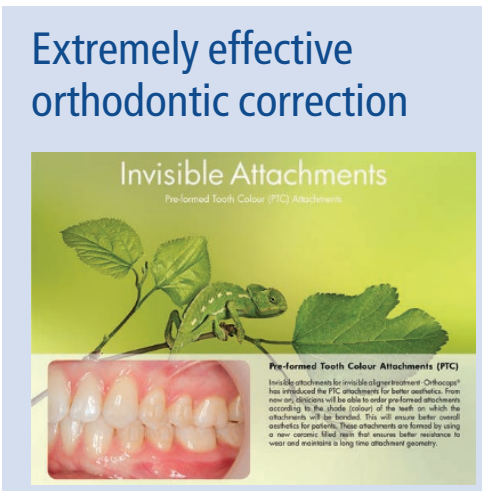

The orthocaps System is an aligner system developed by Dr Wajeeh Khan, an orthodontist practising in Germany. This system has been successfully used for over ten years by 2,000 orthodontists and dentists in 35 countries around the world.

The system has a unique method of combining two different soft aligners for day and night time use to achieve unparalleled results. These two types differ from one another in both their composition and the amount of pressure they exert. This innovation is called the TwinAligner system.

orthocaps aligners are manufactured with high-performance materials and cutting-edge techniques. The orthocaps TwinAligner system represents an important step forward, safety-wise, aesthetically, and technologically, in the field of orthodontics. It employs a hybrid technique of using lingual bracket auxiliaries for complex movements together with aligners to obtain excellent results.

www.orthocaps.com fluoride content of 5\% (22,600 ppm fluoride) Profluorid Varnish protects teeth against acid attack, promotes remineralisation and contributes to the formation of fluorapatite.

With a smooth consistency and excellent adhesion even to moist surfaces, Profluorid Varnish is highly regarded for its excellent time-saving handling and aesthetic properties. Available in five different flavours, including new bubble-gum flavour, Profluoride Varnish appeals to both adults and children and is ideal for treating cervical areas after professional cleaning and calculus removal.

VOCO Profluorid Varnish is available in a choice of multi-application tubes and cartridges, as well as a popular, convenient and patented SingleDose blister pack, supplied ready with an application brush. Change your game today and embrace the 'Teeth for Life' evolution.

To find out more call the expert VOCO team today free on 0080044 444555 or email service@voco.de.

www.voco.com.
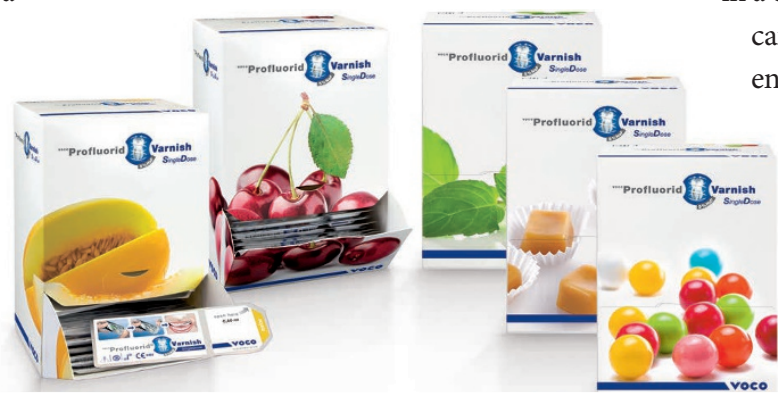

\section{Accelerated healing of traumatised oral tissue}

Dent-O-Care are pleased to announce the launch of Gengigel Spray, a new addition to the Gengigel range. Gengigel is a patented formulation containing high molecular weight hyaluronan, a natural physiological constituent produced in the body to heal and repair itself. Gengigel is widely endorsed by periodontists and hygienists due to its ability to calm inflammation and promote tissue healing. This exciting product range provides opportunities to better manage traumatised oral conditions, such as xerostomia, mouth ulcers, burning mouth syndrome, lichen planus, as well as periodontal disease that is resistant to resolution.

Gengigel is the first completely natural topically applied oral care product to

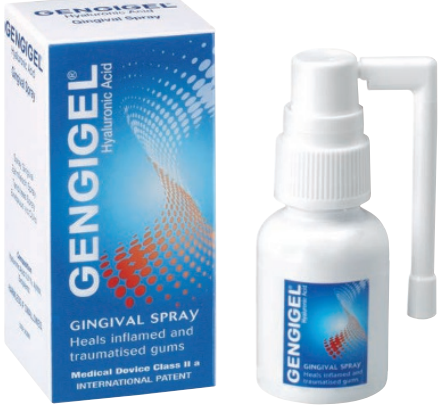

help control the host response to manage all sorts of intra-oral inflammatory and painful conditions, as well as patients with periodontal disease that demonstrate poor healing after treatment.

For more information call 0208459 7550 or email marketing@dentocare.co.uk.

\section{In support of soft tissue management}

Johnson \& Johnson, the makers of LISTERINE, are delighted to present Professor Nicola West speaking on the topic of soft tissue management at this year's British Dental Conference.

Professor West, Head of Periodontology, Professor and Honorary Consultant in Restorative Dentistry at the University of Bristol, will cover topics including managing the soft tissue health of your patients now, securing their future health, and delivering this within the constraints and demands of running a successful dental practice.

Delegates may attend Professor West's presentation on Thursday 25 May at 14:1515:15 or Friday 26 May at 11:45-12:45.

Johnson \& Johnson are the makers of LISTERINE which has variants that are suitable for daily use as an adjunct to mechanical cleaning to help deliver an optimised daily prevention regimen. In addition the LISTERINE Advanced Defence range is available to help dental professionals deliver advanced treatment outcomes for patients.

For further information, visit stand C13 to speak to a member of the LISTERINE Professional team. 